\title{
External Cephalic Version in Cases of Imminent Delivery at Preterm Gestational Ages: A Prospective Series
}

\author{
Meghan G. Hill, MBBS ${ }^{1}$ Kathryn L. Reed, MD ${ }^{1}$ \\ ${ }^{1}$ Department of Obstetrics and Gynecology, Division of Maternal- \\ Fetal Medicine, The University of Arizona, Tucson, Arizona \\ Am J Perinatol Rep 2019;9:e384-e388.
}

\begin{abstract}
Address for correspondence Meghan G. Hill, MBBS, Department of Obstetrics and Gynecology, Division of Maternal-Fetal Medicine, The University of Arizona, 1501 North Campbell Avenue, Tucson, AZ 85724 (e-mail: meghanhill@obgyn.arizona.edu).
\end{abstract}

\author{
Abstract \\ Keywords \\ - preterm \\ - external cephalic \\ version \\ - preterm labor \\ - preterm birth \\ - breech
}

Objective When delivery of a breech fetus is required at a preterm gestational age, Cesarean delivery is often recommended. We performed a prospective patient series to assess the success rate and safety of performing external cephalic version (ECV) procedures on preterm fetuses as an alternative to Cesarean delivery.

Study Design We performed a prospective clinical series of patients who required delivery with a malpresenting fetus at a preterm gestational age.

Results ECV procedures were successful in singletons $50 \%$ of the time. No significant complications or cases of fetal mortality were documented.

Conclusion ECV at preterm gestational ages may be an appropriate approach to management in patients requiring delivery. Larger series are needed to further document success rates and risks of the procedure.

Preterm delivery complicates $12 \%$ of births in the United States. ${ }^{1}$ Preterm fetuses often present with malpresentation. Most of these malpresentations are breech in nature, but less commonly, transverse and oblique lie can be seen. The rates of breech presentation decrease from 20 to $30 \%$ at 25 weeks to 1 to $6 \%$ at term. ${ }^{2-4}$

At an estimated fetal weight of $<2,500 \mathrm{~g}$ with a fetal malpresentation, the standard of care is a Cesarean delivery as fetal complications are higher with vaginal breech delivery under this birth weight. ${ }^{5}$ Additionally, guidelines recommend external cephalic version (ECV) only after 37 weeks of gestation. This leaves patients requiring preterm delivery of a malpresenting fetus $<2,500 \mathrm{~g}$ with the only recommended option for delivery being a Cesarean delivery.

Cesarean deliveries, though performed for fetal benefit in this circumstance, unfortunately do not eliminate the risk to the fetus. ${ }^{6-11}$ The breech fetus delivered via Cesarean accrues an elevated risk of birth trauma compared with its cephalicpresenting counterpart ${ }^{6,7}$. Several studies have questioned the magnitude of benefit of Cesarean delivery to the premature malpresenting fetus. ${ }^{7,12}$

received

June 23, 2019

accepted after revision

October 15, 2019
DOI https://doi.org/

10.1055/s-0039-3401800. ISSN 2157-6998.
Maternal risks of Cesarean delivery as compared with vaginal delivery are well documented. Immediate risks include greater blood loss, thrombotic events, unplanned hysterectomy, operative damage to organs, mortality, longer hospital stay with a higher cost, and more readmissions than in patients undergoing vaginal delivery. ${ }^{13-16}$ The risk of morbidity with Cesarean is approximately three times that with vaginal delivery. ${ }^{17}$ Pregnancy-related risks include repeat Cesareans, placental abnormalities, peripartum hysterectomy, and uterine rupture, with fetal and maternal demise being more frequent, and overall cost of care being higher. ${ }^{16,18}$ Life-long complications include scarring, chronic pain, and intestinal obstruction from adhesive disease. Pregnancies following a Cesarean delivery have a higher rate of placental abnormalities which may lead to preterm birth, unexplained stillbirth, as well as repeat Cesarean delivery, the risks of which include higher rates of neonatal respiratory insufficiency and neonatal intensive care unit admission. ${ }^{19}$ These factors are all concerning, considering the increasing rates of Cesarean delivery. ${ }^{20}$

An option to mitigate the risks in malpresenting fetuses is an ECV. ${ }^{21}$ This procedure has been rigorously studied and has

Copyright @ 2019 by Thieme Medical Publishers, Inc., 333 Seventh Avenue, New York, NY 10001, USA. Tel: +1(212) 584-4662.
License terms

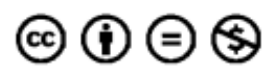


an acceptable success rate when performed at term. ${ }^{22-28}$ Various techniques are used including manipulation of the fetus into a "forward" or "backward" roll to achieve a cephalic presentation. $^{29,30}$

The frequency of breech presentation is the highest in preterm gestations and decreases as a pregnancy approaches term. $^{2-4,31}$ ECV was commonly practiced in an outpatient setting as a part of routine antenatal care between 24 and 41 weeks in the past. In fact, one author commented that an attempt at version should be made before 28 weeks. ${ }^{32}$ Case series have shown extremely low complication rates with multiple ECV procedures being performed in one pregnancy at preterm gestational ages (meaning the patient represented to their next antenatal appointment with a breech fetus which was again, manipulated into cephalic presentation). ${ }^{33}$ Success rates appear to be higher in preterm than term gestations, at least in a nonlabored setting, with 50 to $70 \%$ of fetuses verting to cephalic presentations. ${ }^{32-34}$ However, the utility of this procedure in preterm fetuses has been questioned, as there is a significant rate of reversion to a breech presentation at preterm gestational ages. Moreover, most fetuses will spontaneously assume a cephalic presentation by term, and the procedure is not without risk. Placental abruption, fetal injury (including fractures and brachial plexus injuries), and stillbirth are known complications of the procedure. ${ }^{33}$ The absolute risk of all complications is $1 \%$ in the term fetus, hence offering an ECV remains the standard of care in these gestations. ${ }^{21}$

ECV in the setting of preterm labor, preterm premature rupture of membranes, or indicated deliveries for preterm infants has not been adequately studied. We set out to prospectively study if there was value in the attempt at ECV in pregnancies that needed to be delivered at preterm gestational ages. We also sought to assess the safety of the approach.

\section{Methods}

The University of Arizona Institutional Review Board approved the study (1703269951).

The initial plan had been to compare patients who agreed to participate in the ECV procedure versus those who agreed to data collection only but not an attempt at ECV. However, it became clear that this approach was not feasible because of the low number of interested patients. Hence, we report here only on patients who desired inclusion with an attempt at ECV. The information is presented as a prospective series.

Inclusion criteria were gestational age between 23 and 36 weeks, live fetus or fetuses, presentation other than cephalic (breech, transverse, or oblique), category I or II fetal heart rate monitoring, indication for delivery being (1) labor, (2) preterm prelabor rupture of membranes (with deepest vertical pocket $\geq 2 \mathrm{~cm}$ and cervix dilated $\leq 2 \mathrm{~cm}$ via visual inspection), and (3) maternal requirement for delivery, based on the recommendation of the clinical provider. Use of tocolytic agents were permitted, but not required or standardized in the protocol. Exclusion criteria were fetal demise, placenta previa, or invasive placentation, monoamniotic twin gestation, abnormalities in fetal health assessment, or other contraindication to vaginal delivery.
Patients, who enrolled in the study, were taken to the operating room and administered regional anesthesia for Cesarean delivery. An attempt at ECV was undertaken. If the procedure was successful, the patient was transferred back to a laboring room to proceed with a normal delivery. If the attempt was unsuccessful, a Cesarean delivery was performed.

\section{Results}

Fifteen women agreed to participate in the study. Two women never underwent study procedures, and one woman agreed only to records review (as specified initially in the protocol, the comparator group was abandoned soon after the study was opened). The remaining 12 women underwent study procedures, with 2 having twin gestations and 10 having singleton gestations. Their details are presented in - Table 1 .

Two patients experienced fetal heart rate decelerations after ECV procedures, which is not unexpected and is a known phenomenon after an ECV. Two patients experienced rupture of membranes, one during the ECV procedure, and another on the day of the procedure but not during the ECV procedure. There were no occurrences of immediate maternal or neonatal severe morbidity or mortality related to study procedures.

\section{Discussion}

We have shown in our series that ECV under controlled conditions at preterm gestational ages may be an appropriate alternative to outright Cesarean delivery. We did not document any negative physical outcomes to attempting to vert a preterm fetus in a setting where the clinician is prepared to immediately perform a Cesarean. However, we do note that the rupture of membranes occurred during one attempt and shortly after an ECV procedure in a second patient, indicating that it may be judicious to perform these procedures in the operating room in laboring patients. In our series, the main detractor of the approach is the main detractor of term ECVs, failure of the procedure to provide a cephalic presentation and an ensuing Cesarean delivery.

Our success rate of the ECV was 50\% in singleton pregnancies, which is within the expected rate reported in previous studies in nonlaboring preterm patients. ${ }^{32-34}$ Two studies reported success rates in excess of $70 \%,{ }^{34,35}$ which we did not achieve and suspect our study design may be responsible for this. One issue is that the patients involved in this study had their ECV procedures in the operating room (OR) with anesthetic administered (with the exception of one patient with a protocol violation). If the procedure failed, a Cesarean was then performed. We believe that this was an ethical approach; however, it meant that patients, who were likely to deliver, did not have their ECV procedures until the clinician was absolutely sure about a delivery needed to be performed. Hence, 9 of the 12 patients were in advanced labor (which we consider to be $\geq 4 \mathrm{~cm}$ dilation) with a wellengaged fetal part when study procedures were initiated.

We did not document harm to fetuses or mothers attributable to the procedure. The neonates with the lowest Apgar scores were those that were the earliest in gestational ages, 


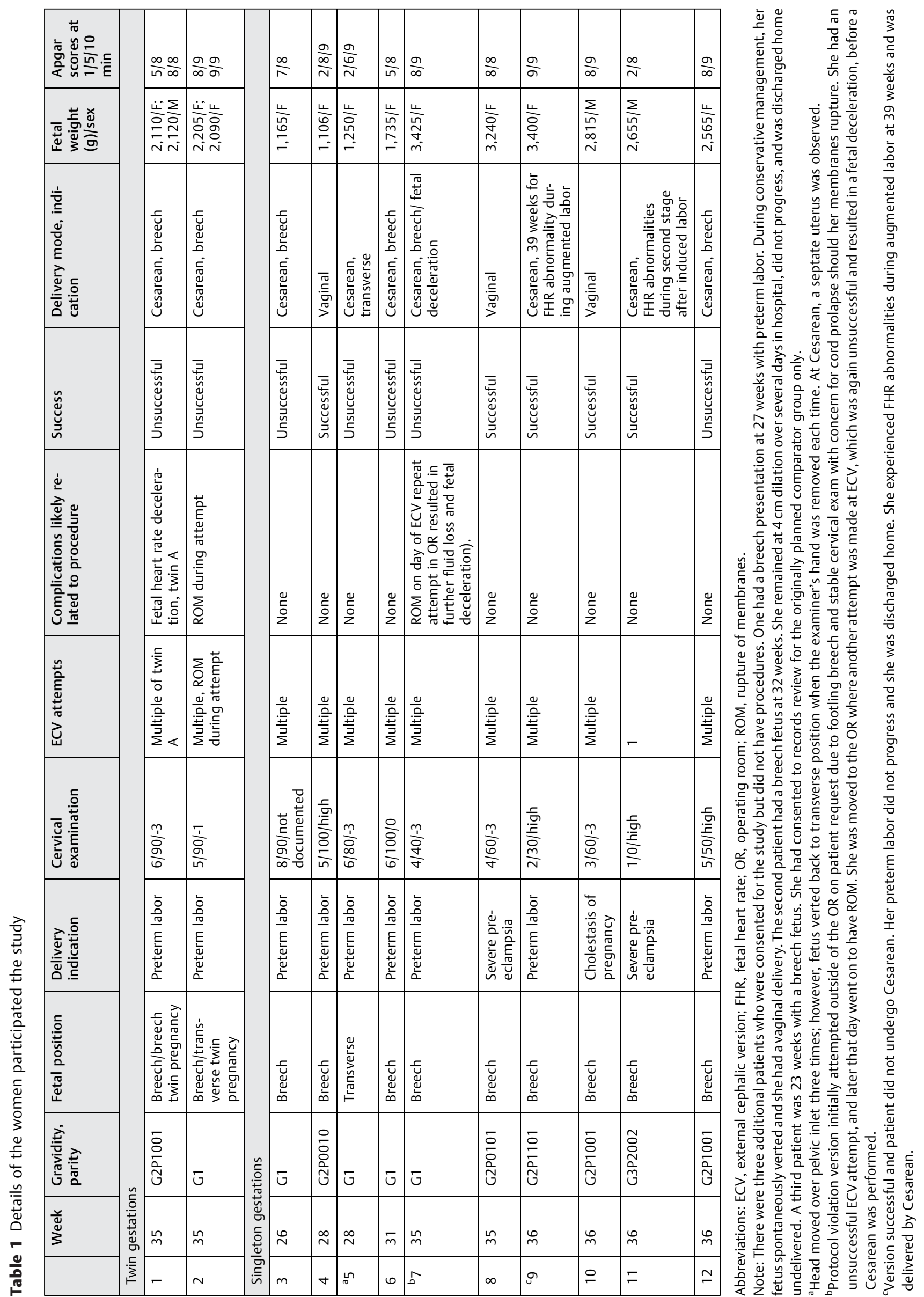


suggesting an effect of prematurity rather than the ECV procedure. This is not surprising considering the compressive forces that we know a fetus can endure for delivery either through a pelvis or a Cesarean incision. As a surrogate for $\mathrm{ECV}$, we refer to the evidence base for operative delivery in the preterm fetus which requires compressive forces to the fetus to effect vaginal delivery. Arguably, obstetric forceps most closely mimic the external force to the fetal head as seen in the preterm ECV. Forceps are the standard of care for instrumental delivery of the preterm fetus below 34 weeks. In a comparison of Cesarean delivery, vacuum and forcep deliveries, the highest risk of adverse neurologic outcomes is found in the Cesarean and vacuum deliveries ${ }^{36,37}$ though all three methods are commonly used in the delivery of preterm infants. ${ }^{38}$

Term ECV is usually performed before the onset of labor. However, studies have shown it to be a safe procedure for term women in labor, women with ruptured membranes, and a cervix without advanced dilatation, in twin gestations where the first fetus is not vertex, in patients in which body habitus makes Cesarean delivery challenging, and in patients with a low volume of amniotic fluid. ${ }^{23,35,39-42}$ Even with an open cervix, in a laboring patient, no incidences of rupture of membranes occurred in the studies identified in our literature review. ${ }^{35,39-42}$ Several studies have investigated the role to injection of saline into the uterus to facilitate versions, a procedure which increases intra-amniotic pressures, without the occurrence of rupture of the membranes. ${ }^{43,44}$ We did encounter the rupture of membranes in two patients, though it did not appear to lead to harm as these patients were already going to be delivered by Cesarean if their ECV procedures were not successful.

Two of the patients with successful ECV procedures went on to require Cesarean delivery, both of them for fetal heart rate abnormalities in labor. The first patient underwent Cesarean during the second stage and the second underwent Cesarean during the first stage of labor several weeks later. Hence, we believe the Cesareans performed were likely unrelated to the ECV procedures.

Our work has several weaknesses. The most obvious is the small number of patients. Preterm deliveries often occur emergently, outside of usual working hours, when an investigator was not available to consent for study procedures. Several patients with HELLP syndrome demonstrating elevated liver enzymes, who were technically eligible for the study, were not approached for inclusion. As investigators, we were concerned about applying pressure to the abdomen where there appeared to be an imminent risk of liver capsule rupture. Moreover, performing the ECV procedure in the studied population is controversial and hence a challenge. However, we believe this small series of patients shows encouraging results and may provide the foundation for a larger study.

Another shortcoming is the lack of follow-up data. Though there did not appear to be immediate, permanent damage to any infants or women, the true test of this is long-term follow-up. This would be difficult to compare in any population as preterm infants are at an increased risk of neurological injury based on their early gestational age at delivery.
In conclusion, we are encouraged by our findings in this series of patients. Under controlled conditions, we were able to prevent primary Cesareans in several women with preterm breech fetuses without an apparent risk to the women or their infants. This is unsurprising considering there is much evidence for the safety of the procedure at preterm gestational ages, albeit the evidence being cited is from antepartum outpatient care, rather than in laboring patients or those who are then to be induced. We would like to see larger series of patients to confirm the safety of the approach.

Precis

External cephalic version may be an appropriate procedure to offer as an alternative to Cesarean delivery at preterm gestational ages.

\section{Funding}

The authors have no financial support to disclose.

\section{Conflict of Interest}

None declared.

\section{References}

1 American College of Obstetricians and Gynecologists Committee on Practice Bulletins - Obstetrics, Practice bulletin no. 130: Prediction and Prevention of Preterm Birth. Obstet Gynecol 2012;120(04):964-973

2 Hill LM. Prevalence of breech presentation by gestational age. Am J Perinatol 1990;7(01):92-93

3 Hickok DE, Gordon DC, Milberg JA, Williams MA, Daling JR. The frequency of breech presentation by gestational age at birth: a large population-based study. Am J Obstet Gynecol 1992;166(03): 851-852

4 Scheer K, Nubar J. Variation of fetal presentation with gestational age. Am J Obstet Gynecol 1976;125(02):269-270

5 American College of Obstetricians and Gynecologists, Committee Opinion No. 745, Mode of Term Singleton Breech Delivery. Obstet Gynecol 2018;132(02):531-532

6 O'Callaghan ME, MacLennan AH, Gibson CS, et al; Australian Collaborative Cerebral Palsy Research Group. Epidemiologic associations with cerebral palsy. Obstet Gynecol 2011;118(03): 576-582

7 Alexander JM, Leveno KJ, Hauth J, et al; National Institute of Child Health and Human Development Maternal-Fetal Medicine Units Network. Fetal injury associated with cesarean delivery. Obstet Gynecol 2006;108(04):885-890

8 Smith JF, Hernandez C, Wax JR. Fetal laceration injury at cesarean delivery. Obstet Gynecol 1997;90(03):344-346

9 Aburezq H, Chakrabarty KH, Zuker RM. Iatrogenic fetal injury. Obstet Gynecol 2005;106(5 Pt 2):1172-1174

10 Anderson GD, Bada HS, Shaver DC, et al. The effect of cesarean section on intraventricular hemorrhage in the preterm infant. Am J Obstet Gynecol 1992;166(04):1091-1099, discussion 1099-1101

11 Malloy MH, Onstad L, Wright E; National Institute of Child Health and Human Development Neonatal Research Network. The effect of cesarean delivery on birth outcome in very low birth weight infants. Obstet Gynecol 1991;77(04):498-503

12 Richmond JR, Morin L, Benjamin A. Extremely preterm vaginal breech delivery en caul. Obstet Gynecol 2002;99(06):1025-1030

13 Liu S, Heaman M, Joseph KS, et al; Maternal Health Study Group of the Canadian Perinatal Surveillance System. Risk of maternal postpartum readmission associated with mode of delivery. Obstet Gynecol 2005;105(04):836-842 
14 Krebs L, Langhoff-Roos J. Elective cesarean delivery for term breech. Obstet Gynecol 2003;101(04):690-696

15 Bodelon C, Bernabe-Ortiz A, Schiff MA, Reed SD. Factors associated with peripartum hysterectomy. Obstet Gynecol 2009;114 (01):115-123

16 Lydon-Rochelle M, Holt VL, Easterling TR, Martin DP. Cesarean delivery and postpartum mortality among primiparas in Washington State, 1987-1996(1). Obstet Gynecol 2001;97(02):169-174

17 Liu S, Liston RM, Joseph KS, Heaman M, Sauve R, Kramer MS; Maternal Health Study Group of the Canadian Perinatal Surveillance System. Maternal mortality and severe morbidity associated with low-risk planned cesarean delivery versus planned vaginal delivery at term. CMAJ 2007;176(04):455-460

18 Lydon-Rochelle M, Holt VL, Easterling TR, Martin DP. Risk of uterine rupture during labor among women with a prior cesarean delivery. N Engl J Med 2001;345(01):3-8

19 Smith GCSPJ, Pell JP, Dobbie R. Caesarean section and risk of unexplained stillbirth in subsequent pregnancy. Lancet 2003;362 (9398):1779-1784

20 Ford J, Grewal J, Mikolajczyk R, Meikle S, Zhang J. Primary cesarean delivery among parous women in the United States, 1990-2003. Obstet Gynecol 2008;112(06):1235-1241

21 American College of Obstetricians and Gynecologists' Committee on Practice Bulletins-Obstetrics. Practice bulletin no. 161: external cephalic version. Obstet Gynecol 2016;127(02):e54-e61

22 Cook HA. Experience with external cephalic version and selective vaginal breech delivery in private practice. Am J Obstet Gynecol 1993;168(6, Pt 1):1886-1889, discussion 1889-1890

23 Ferguson JE II, Armstrong MA, Dyson DC. Maternal and fetal factors affecting success of antepartum external cephalic version. Obstet Gynecol 1987;70(05):722-725

24 Donald WL, Barton JJ. Ultrasonography and external cephalic version at term. Am J Obstet Gynecol 1990;162(06):1542-1545, discussion 1545-1547

25 Dyson DC, Ferguson JE II, Hensleigh P. Antepartum external cephalic version under tocolysis. Obstet Gynecol 1986;67(01):63-68

26 Boucher M, Bujold E, Marquette GP, Vezina Y. The relationship between amniotic fluid index and successful external cephalic version: a 14-year experience. Am J Obstet Gynecol 2003;189 (03):751-754

27 Newman RB, Peacock BS, VanDorsten JP, Hunt HH. Predicting success of external cephalic version. Am J Obstet Gynecol 1993; 169(2, Pt 1):245-249, discussion 249-250

28 Dugoff L, Stamm CA, Jones OW III, Mohling SI, Hawkins JL. The effect of spinal anesthesia on the success rate of external cephalic version: a randomized trial. Obstet Gynecol 1999;93(03): 345-349

29 Ranney B. The gentle art of external cephalic version. Am J Obstet Gynecol 1973;116(02):239-251

30 Zhang J, Bowes WA Jr, Fortney JA. Efficacy of external cephalic version: a review. Obstet Gynecol 1993;82(02):306-312

31 Luterkort M, Persson PH, Weldner BM. Maternal and fetal factors in breech presentation. Obstet Gynecol 1984;64(01):55-59

32 Macarthur JL. Reduction of the hazards of breech presentation by external cephalic version. Am J Obstet Gynecol 1964;88:302-306

33 Hibbard LT, Schumann WR. Prophylactic external cephalic version in an obstetric practice. Am J Obstet Gynecol 1973;116(04): 511-518

34 Kornman MT, Kimball KT, Reeves KO. Preterm external cephalic version in an outpatient environment. Am J Obstet Gynecol 1995; 172(06):1734-1738, discussion 1738-1741

35 Ferguson JE II, Dyson DC. Intrapartum external cephalic version. Am J Obstet Gynecol 1985;152(03):297-298

36 Werner EF, Janevic TM, Illuzzi J, Funai EF, Savitz DA, Lipkind HS Mode of delivery in nulliparous women and neonatal intracranial injury. Obstet Gynecol 2011;118(06):1239-1246

37 FDA Public Health Advisory: Need for caution when using Vacuum Assisted Delivery Devices. U.S. Department of Health and Human Services. Available at: https://www.thefreelibrary.com/FDA+ Public +Health+Advisory\%3A+Need +for +Caution+when+Using +Vacuum...-a056456763. Accessed November 19, 2019

38 Morales R, Adair CD, Sanchez-Ramos L, Gaudier FL. Vacuum extraction of preterm infants with birth weights of 1,500-2,499 grams. J Reprod Med 1995;40(02):127-130

39 Chervenak FA, Berkowitz RL. Successful external cephalic version in a massively obese patient. Obstet Gynecol 1983;62(3, Suppl):8s-9s

40 Phelan JP, Stine LE, Edwards NB, Clark SL, Horenstein J. The role of external version in the intrapartum management of the transverse lie presentation. Am J Obstet Gynecol 1985;151(06): 724-726

41 Bloomfield MM, Philipson EH. External cephalic version of twin A. Obstet Gynecol 1997;89(5, Pt 2):814-815

42 Brost BC, Adams JD, Hester M. External cephalic version after rupture of membranes. Obstet Gynecol 2000;95(6, Pt 2):1041

43 Benifla JL, Goffinet F, Darai E, Madelenat P. Antepartum transabdominal amnioinfusion to facilitate external cephalic version after initial failure. Obstet Gynecol 1994;84(06):1041-1042

44 Adama van Scheltema PN, Feitsma AH, Middeldorp JM, Vandenbussche FP, Oepkes D. Amnioinfusion to facilitate external cephalic version after initial failure. Obstet Gynecol 2006;108(3, Pt 1):591-592 